\title{
XVI. On brass wind instruments as resonators
}

\section{D.J. Blaikley}

To cite this article: D.J. Blaikley (1878) XVI. On brass wind instruments as resonators , Philosophical Magazine Series 5, 6:35, 119-128, DOI: 10.1080/14786447808639484

To link to this article: http://dx.doi.org/10.1080/14786447808639484

$$
\text { 曲 Published online: } 13 \text { May } 2009 .
$$

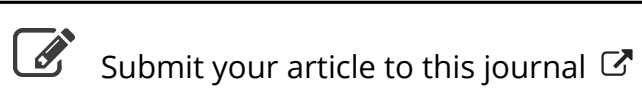

\footnotetext{
Џll Article views: 3
}

Q View related articles $\sqsubset$ 
thinner wires, and, probably owing to the high tension of the former, faint sounds were more readily transmitted: thus the accidental or intentional touching of the tuning-fork with the rim of the mouth-piece, causing a slight clicking sound, was distinctly heard through the ear-piece at a distance of 150 yards-and this, even although the two attachments of copper wire were practically at right angles to the main wire, whereby part of the sound would pass away onwards up and down the line.

6. The great delicacy of the action may be inferred from the fact that fine sand strewn upon the disk of the ear-piece is unaffected by conversation through lengths of about 7 yards. The sensitiveness also of the mouth-piece was shown by sounds not spoken into it being readily transmitted, such as coughing, laughing, or remarks made by persons standing beside the instrument. Indeed, in some cases an advantage is obtained by keeping back from the mouth- or ear-pieces; and the author has sometimes thought an improvement was obtained by holding the ear-piece slightly inclined to the ear.

In all cases the individual voice could easily be distinguished though modified more or less by the structure and material of the mouth- and ear-pieces.

The mouth- and ear-pieces were usually of the same form and material, and were therefore used for either speaking or hearing. Some forms, however, do better as ear-pieces, others as mouth-pieces.

In conclusion, the author believes that many interesting physical questions may be studied by means of these arrangements, and that practical application may be made where communication of this nature is required.

\section{On Brass Wind Instruments as Resonators.} By D. J. BLAikLeY*.

[Plate I.]

TN bringing before the Physical Society a few notes and 1 experiments on this subject, I would desire to say that they are the result of an attempt to carry somewhat more into detail than, as far as I am aware, has hitherto been done, some aconstical investigations of the late Sir C. Wheatstone. A most interesting paper on Wheatstone's work in this field was brought before the Musical Association by Professor W. G. 1878.

* Communicated by the Physical Society, having been read May 25, 
Adams in 1876; and to that paper I am in great measure indebted.

A brass instrument may be defined as a resonator capable of reinforcing a certain fundamental periodic vibration originated by the lips, and all such vibrations as have for their relative numbers $2,3,4, \& c$. when the fundamental note is represented by unity, - these vibrational numbers being the basis of what is known as the natural harmonic series of musical intervalsand this series being the same, whaterer may be the absolute pitch of the fundamental note or the character of tone of the instrument.

It is possible to make the lips give notes which, although scarcely audible, are of definite pitch, without the use of an instrument, just as a tuning-fork gives its proper note with or without a resonator.

There are two simple forms of resonators which give the series of notes required in wind instruments: these are the open tube of equal section throughout, and the cone complete to its apex, where it is of course closed. In the tube the warelength of any note is inversely proportional to its ribrational number; and the nodes or points of maximum compression and rarefaction, and the centres of the ventral segments, or points of maximum amplitude of vibration are equidistant: but in the cone this is not the case. Wheatstone found experimentally that the notes of a closed cone agree in pitch with those of an open tube of the same length; and therefore the prime or fundamental tone of such a cone, is an octave higher than the prime of a closed tube of the same length. He found also that in conic frustra of similar lengths, but of different proportions as regards the diameters of their ends, the pitch varied, rising as the difference between the two ends increased when the small end was closed, and becoming lower under the same conditions when the large end was closed. The accompanying diagram (Plate I.) shows the positions of the nodes and centres of ventral segments in an open tube and a cone of the same length for the notes $c, c^{\prime}, g^{\prime}, c^{\prime \prime}$, marked $1,2,3,4$ ( $c$ having 128 vib., and a wave-length of 105 in. at $60^{\circ} \mathrm{F}$.). The numerals grouped together and marked $\mathrm{N}$ show the positions of the nodal points or surfaces, and those marked $\frac{V . S}{2}$ the centres of the ventral segments or points of maximum vibration. The effect that the diminishing size of the cone has upon the position of the nodes may be easily traced. Whilst the positions of the centres of the ventral segments remain the same as in the open tube (the numerals for these on the cone in the diagram falling exactly under those for the open tube), the nodes 
Phil Mas. S 5.Vol. 6. Pl. I.
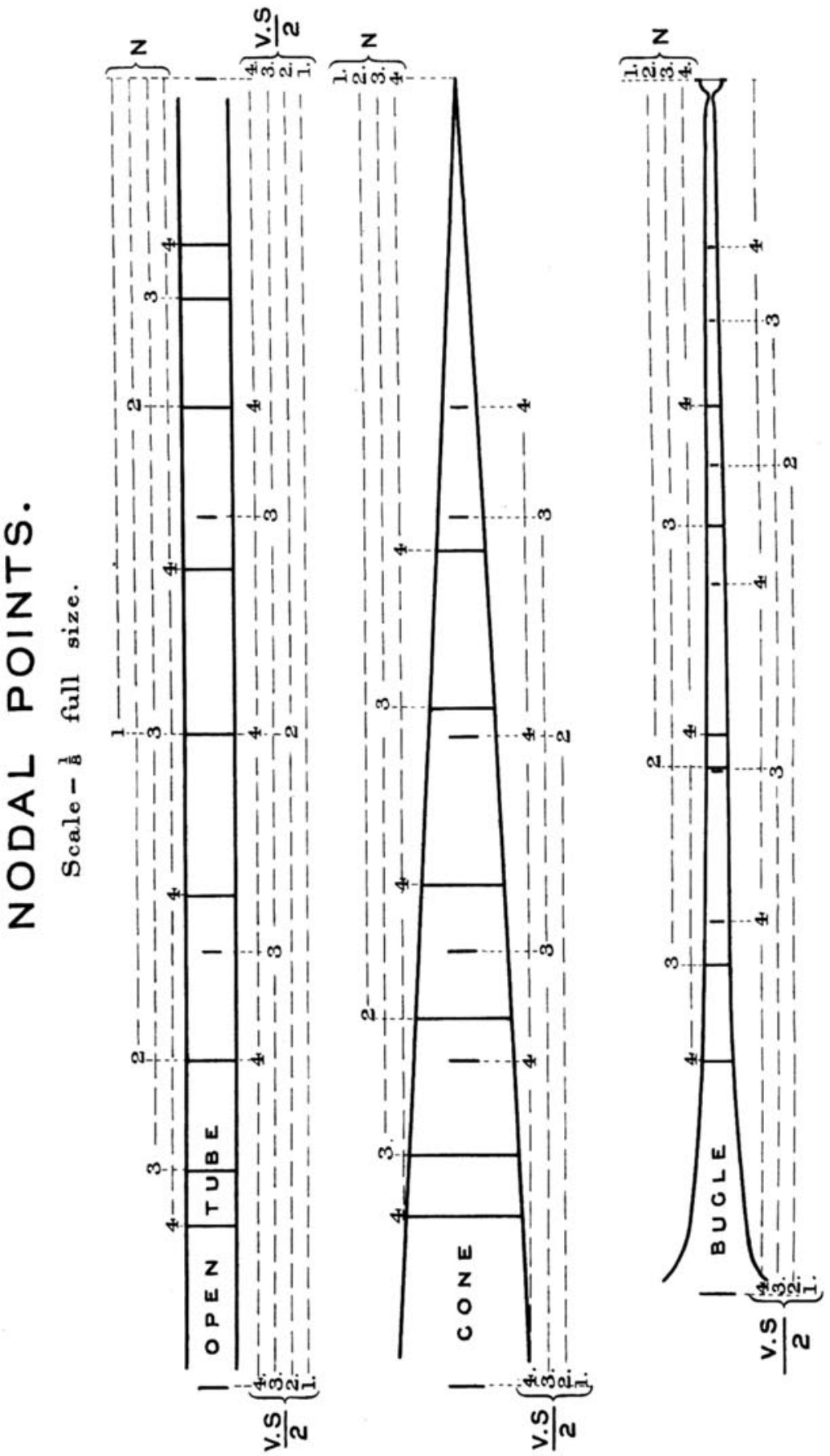
are gradually further and further apart, dividing their respective ventral segments more and more unequally, until at the apex of the cone is a node common to all the notes. It follows from this that the centre of a ventral segment in a cone is not the centre of the length between its nodes, and, conversely, that as the diameters of the two ends of the ventral segment approach equality, so does the position of the node become more central, until the condition of vibration existing in an open cylindrical tube is reached; and such a tube may evidently be considered as a portion of a cone whose apex is at an infinite distance. It is to be noticed that in the cone the number of $\frac{1}{4}$ wave-lengths, or semi- ventral segments, is not directly proportional to the vibrational number as in the open tube, but, with the exception of the fundamental note, is always in excess. Thus let

then

$$
\begin{aligned}
\mathrm{N} & =\text { number of } \frac{1}{4} \text { wave-lengths, } \\
n & =\text { relative vibrational number } ;
\end{aligned}
$$

$$
\mathrm{N}=n+(n-1)=2 n-1 \text {. }
$$

Instances.-Note 1 (fundamental) $\mathrm{N}=1+(1-1)=1$,

Note 4 (double octave) $\mathrm{N}=4+(4-1)=7$.

The velocity of the portion of wave or waves in the cone therefore differs with the pitch of the note, and is in no case the same as the velocity in free space. Assuming this latter to be 1120 feet per second, we should have in the cone the following velocities :-

$\begin{array}{llllcl}\text { Note. } & & n . & \text { N. } & \begin{array}{l}\text { Feet per } \\ \text { second. }\end{array} \\ c & 128 & \ldots \ldots & 1 & 1 & 2240 \\ c^{\prime} & 256 & \ldots \ldots & 2 & 3 & 1493 \cdot 4 \\ g^{\prime} & 384 & \ldots \ldots & 3 & 5 & 1344 \\ c^{\prime \prime} & 512 & \ldots \ldots & 4 & 7 & 1280\end{array}$

and the space traversed by the waves of the different notes in one second, measuring from the apex of the cone to, say, the ear of an observer:-

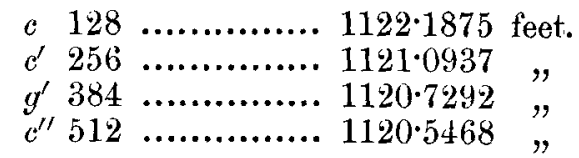

The method I used to find the positions of the nodal points in the cone, and which is applicable to wind instruments or tubes of any varying section, may be illustrated by a conic frustum open at both ends. Holding a vibrating fork over one end (in this case $c 512$ ), gradually sink the tube in water: 
the water-level when the tube is giving its maximum resonance shows the position of the node.

Brass instruments are generally considered to be cones, or cones combined with cylindrical tubing, neither of which descriptions properly applies; and this I will endeavour to make clear by experiment. We may, in the first place, consider whether the resonance of cones and tubing is influenced appreciably by the action of the lips; and it will be found that, whether the lips or a tuning-fork be used to excite the vibration, the pitch is the same. Two illustrations may be giventhe first a common hunting-horn, pitch $c 512$ when it is blown, and giving an excellent resonance to the $c 512$ fork when the mouthpiece is closed; if, however, we slightly alter its length either way, the resonance to the fork is no longer at its maximum. For the second illustration, I take a cylindrical tube which becomes closed on being placed against the lips: blowing it as a wind instrument, we find its proper tones are e $128, g^{\prime} 384, e^{\prime \prime} 640, b^{\prime \prime} b 896, \& c$. , the same as it would give as a resonator, and that the pitch of these tones is so definite that it is very difficult to alter any of them by the lips more than two or three vibrations, except the lowest. We may here note that the power of a resonator to reinforce the different notes of a series of tones, with the prime or fundamental one of which it is not truly in unison, is much greater for the fundamental than for the higher notes; and this gives the reason for the ease with which the fundamental note of a wind instrument may be varied within pretty wide limits, say half a tone sharper or flatter than its proper pitch. Taking, for illustration, a closed tube 21 inches long, it will be found to give appreciable resonance to a fork of 128 vibrations with quarter wave-length of $26 \frac{1}{4}$ inches, but scarcely any to a fork of 384 vibrations ( $g^{\prime}$ the twelfth from $\left.c 128\right)$ with quarter wave-length of 83 inches : when the $c$ fork is sounding, the length of the resonance-chamber is to the quarter wave-length as 21 to $26 \frac{1}{4}$; but when the $g$ fork is used, the corresponding proportion is practically as $3 \frac{1}{2}$ to $8 \frac{3}{4}$; for in this case there is a second node at a half wave-length, or $17 \frac{1}{2}$ inches from the closed end.

For musical purposes a cylindrical tube blown by the lips is evidently unsuited, by reason of its poor tone, as well as by its giving only the odd intervals. The cone gives the required intervals; but it cannot be used by the lips in its complete form ; it would be necessary to cut off a considerable portion to get sufficient width for the action of the lips. Assuming the cone shown on diagram to be cut at the second node of note 4 (counting the node at the apex as the first) and there closed by the lips, that note of the original cone can still be 
sounded, but no other; the other notes that can be produced may be regarded as the notes 3 and 2 made flatter by their nodes being drawn back, as it were, to the position of node 4 , where the cone is cut and the lips are placed; the original notes $2,3,4$, or $c^{\prime}, g^{\prime}, c^{\prime \prime}$, becoming thus the 1st, 2nd, and 3rd notes of a new inharmonic series, with pitches approximately $c \sharp, e b^{\prime}, c^{\prime \prime}$-thus approaching the notes of a cylindrical stopped tube. I have here two other tubes tapering in different degrees- the first two proper tones on the one being $c^{\prime}$ and $c^{\prime \prime \prime}$, and on the other $e^{\prime}$ and $e^{\prime \prime}$ (a major tenth). From these experiments it may be seen that, by using portions of cones of different proportions with their small ends closed, it is possible to get different series of intervals varying between those of an open and those of a closed cylindrical tube-that is, the first interval varying between an octave and a twelfth.

One of the examples just shown (the tube with notes $c^{\prime}$ and $\left.e \$^{\prime \prime}\right)$ appears to give intervals not very far removed from those required: it may be made use of to illustrate the effect of the combination of a cone with cylindrical tubing, such tubing being of necessity used in practice in connexion with valves or slides to complete the scale. Flattening this cone a fourth, from $e^{\prime}$ to $g$, by adding tube, it gives the intervals $g, e^{\prime}, d^{\prime \prime}$ in place of the $g, g^{\prime}, d^{\prime \prime}$ required, or the ratios $1,1 \frac{2}{3}, 3$ in place of $1,2,3$, the second interval being actually greater than the first.

These illustrations prove that neither a conic frustum, nor a conic frustum combined with cylindrical tubing, can truly be resonators to notes in the natural harmonic series; but seeing that a bugle or other wind instrument, although it has a considerable diameter at the mouthpiece, may nevertheless be in tune, it appears that its nodal points cannot be in the same positions as those in the cone. On the diagram is represented a bugle of the same pitch as the open tube and cone, with the positions of its nodes and semi- ventral segments as determined by experiment with tuning-forks. Comparing on the diagram the positions of the nodes of any given note in both the bugle and the cone, it will be noticed that there are great differences. The nodes of note 2 show this clearly. Compare lengths from both ends: from mouthpiece to node the length is more nearly equal to that between similar nodes on cylindrical tubing than to that between similar nodes on the cone; but from node to open end it is greater than on the cone, the bugle opening more rapidly.

Thus, then, by altering the proportions of the different semiventral segments of which such an instrument may be conceived to be built up, the positions of the nodes may be so 
arranged that there shall be a node for every note of the harmonic series at the mouthpiece as required; and according as that is more or less perfectly effected will the instrument be more or less perfectly in tune. This bugle is divided into its seven semi-ventral segments for its 4 th note, $c 512$, according to the diagram; and it will be found that by blowing at any one of the nodal points, with any length of the bugle containing an odd number of semi- ventral segments, the note $c^{\prime \prime}$ can be produced. The total number of pieces and combinations that can give this note is eighteen.

Having given these few illustrations of the conditions upon which correct intonation, or the relative pitch of the different notes that can be sounded on a brass instrument, depends, I will now endeavour to show the connexion there is between this point and the question of quality of tone, understanding by quality of tone that characteristic of sound which enables us to recognize a difference between tones of the same pitch. Helmholtz has fully demonstrated that it is only in exceptional cases that we hear a simple musical tone-the vast majority of musical tones being in reality compound tones, in which the fundamental or prime tone has blended with it many upper partial tones of the natural harmonic series, - and that the variety of quality of tone depends mainly upon the number and intensity of these upper partial tones. Blowing the note $c 256$ on three resonators of different forms we get three distinctly different qualities of tone: the resonators now used are a common paraffin-lamp chimney, the conic frustum already shown (having for its first two proper tones $c^{\prime}$ and $c^{\prime \prime} \sharp$ ), and the bugle. Analyzing these three tones by tuning-forks or resonators, we find that the lamp-chimney of irregular form gives no upper partials; the tone is pure or simple. The cone has the second and third partials sounding, but not strongly, as it is not strictly in tune for them, or, in other words, there is a difference of phase between the prime tone and the partials. And the bugle has all the partials up to the seventh, gradually diminishing in power, but all tolerably strong up to the fifth inclusive. Slightly altering the form of the cone by adding tubing to the narrow end, and maintaining the original pitch of the prime tone ( $c 256$ ), by cutting a portion off the wide end the pitch of the second tone may be altered until it is $c 512$, an exact octave from the prime; and we find that the quality of tone of the prime or fundamental note is altered, owing to the more perfect resonance which the cone now gives to its second partial. In its original form, with proper tones $c^{\prime}-c \sharp^{\prime \prime}$, the cone could give but an imperfect resonance to $c^{\prime \prime}$, the second partial to its prime $c^{\prime}$. 
In the trombone and the euphonion we have two instruments of very different and characteristic qualities of tonesthe trombone being brilliant and piercing, and the euphonion mellow. We may take the $\mathrm{Bb}$ of about 120 vibrations (army pitch) on each of these and endeavour to give a visible proof of the existence of high upper partial tones. For this purpose I use small tubular resonators covered at one end with a tightly strained diaphragm or tympanum of goldbeater's skin, against the centre of which is hung a very small bead, or drop of sealing-wax, by means of a single thread of cocoon-silk. The two resonators now used are tuned respectively to the fourth and ninth partials of $\mathrm{Bb}$, or $b^{\prime} b$ of 480 vibrations and $c^{\prime \prime \prime}$ of 1024. When the proper tone of such a resonator is sounded in its neighbourhood, either as a simple tone or as a partial in a compound tone, the agitation of the membrane puts the bead in violent motion, which can easily be seen in the image thrown on the screen by the electric lamp. It will be noticed that when $\mathrm{Bb}$ is sounded, either on the euphonion or on the trombone, both resonators are agitated-but that the excursions of the bead due to the partial tone of 1024 vibrations are much greater with the latter than with the former instrument, although both are played with but moderate force, thus proving that, though partials as high as the ninth exist in the quality of tone produced by both these instruments, yet in the trombone the upper partials have much greater strength than in the euphonion. With a resonator more suitable for private experiment than these, I have distinctly heard the sixteenth partial tone in the $\mathrm{Bb}$ of the trombone. A tapering tube open at both ends, or a common wine-bottle with the bottom knocked out, is very convenient for analyzing tones. Sinking such a tube in water and holding the ear close against the small end, the various partials existing in a given compound tone may be readily discerned, as the length of the tube changes according to the depth it is immersed in the water.

Although one wind instrument may be made to approach another in quality of tone by means of different methods of blowing, and it is therefore not so easy to analyze the tones of these as it is those of keyed instruments with fixed tones, yet I have endeavoured to establish some general data; and these I will lay before you. The instruments the tones of which I have analyzed are the $\mathrm{Bb}$ tenor trombone, the $\mathrm{Bb}$ euphonion, the F French horn, the Bb cornet, and the bugle. The partial tones named in the Table are those heard when the instruments are gently blown; with loud blowing higher tones can be discerned. The ordinary marks of musical expression, $p p$, $p, m f, f$, are added in cases where I found it possible to make a comparison. 


\begin{tabular}{|c|c|c|c|}
\hline Instrument. & Note. & $\begin{array}{l}\text { No. of } \\
\text { vibrations. }\end{array}$ & Partial tones heard. \\
\hline \multirow{4}{*}{$\mathrm{Bb}$ Trombone } & $\mathbf{B}, \mathbf{b}$ & 60 & $1,2,3,4,5,6, \&$ e. to 16 . \\
\hline & $\mathrm{Bb}$ & 120 & $1,2,3,4,5,6$, \&c. to 12 . \\
\hline & $f$ & 180 & $1,2,3,4,5,6,7$ \\
\hline & $B, b$ & 60 & $1,2,3,4,5,6$, \&c. to $12 ;$ higher $^{p}$ \\
\hline \multirow{2}{*}{ Bb Euphonion ... } & $\mathrm{Bb}$ & 120 & $1,2,3,4,5,6,7$ \\
\hline & $f$ & 180 & $1,2,3,4,5$ \\
\hline \multirow{3}{*}{ F French hom ... } & $c$ & 135 & $1,2,3,4,5,6,7,8$ \\
\hline & $f$ & 180 & $1,2,3,4,5,6$. \\
\hline & $f^{\prime}$ & 360 & $1,2,3,4,5,5,6$ \\
\hline Bb Cornet............. & $b b$ & 240 & $1,2,3,4, \stackrel{p}{p}, 6,7,8$ \\
\hline Bugle ................. & $\ldots$ & 256 & $\begin{array}{c}1,2,3,4,5,6,7 \\
f m f p p p\end{array}$ \\
\hline
\end{tabular}

While submitting that the different qualities of tone are accounted for by the difference in the number and force of the upper partials in any given compound tone, I must at the same time acknowledge that I can do no more than throw out a few suggestions with respect to the causes that influence the production of such uppor partials in this remarkable manner. The partials being in the natural harmonic series, it is evident that if the various proper tones of a vibrating column of air such as is enclosed in a wind instrument are not in exact agreement with this series, the resonance to the partials cannot be at its best. Take for illustration two instruments nominally the same (say two bugles), but with somewhat different qualities of tone. Suppose that a certain compound tone on both should have its first and second partials of equal intensity, but that one instrument has that one of its proper tones that is nearest in pitch to the required second partial a semitone sharper than that partial; the supposed compound tone sounded on that instrument will be deficient in the quality the second partial should give.

As regards instruments of different characters, the chief points influencing the tone are the general form of the instrument (understanding by this the proportions of the column of air, and not the shape into which the instrument may be bent up for the convenience of the player), the extent of the flanging of the bell, and the form of the mouthpiece. As an 
illustration of the first of these conditions the trombone may be compared with the euphonion; the tubing of the trombone is cylindrical for about two thirds of its length from the mouthpiece, but the euphonion opens with gradually increasing curvature from the mouthpiece to the rim of the bell. The high upper partials being more powerful on the former than on the latter instrument, it would appear that the cylindrical tubing has the power of maintaining the intensity of the short waves to a greater extent than the tapering tubing has. The bellflange may be increased in size to a considerable degree without altering the pitch of an instrument; but such increase has a marked effect on the quality of tone, greatly subduing the force of the upper partials. I find by experiment that the pitch is not altered by the extension of the flange curvature beyond a point at which its tangent would make an angle of about $40^{\circ}$ with the axis of the instrument, although the quality of tone is decidedly altered by such extension. This may be illustrated by changing the bell-end of a bugle for a bell with much wider flange, more like that of a French horn: comparing the two, it will be noticed that the change in quality of tone is very marked.

The form of the cup of the mouthpiece varies for different instruments, from that of a long deep conical funnel to that of a comparatively shallow well-rounded cup-the first form representing the French-horn mouthpiece, and the second the mouthpiece for instruments of brilliant tone, as the trumpet and trombone; those for cornets, bugles, and saxhorns are of an intermediate character. Although it is manifest that a shallow cupped mouthpiece favours the production of high upper partials, I have not as yet succeeded in arranging any experiments which would illustrate the cause of this. One fact, however, noticed by many observers, appears to me to be suggestive, and worth bearing in mind in connexion with this subject. It is this:-If a vibrating tuning-fork be placed on a sounding-board, the quality of tone it gives varies with the pressure applied: touching the board very lightly with the fork the prime tone is well heard; but on pressing the fork down to the board the tone appears to jump up an octave; at least the second partial (octave of the prime) is heard with great distinctness. This experiment appears to prove that if an elastic resonant body (in this case the resonant board) is in a state of initial pressure at the point of origin of vibrations, a vibration that would otherwise be simply pendular becomes a vibration compounded of two or more simple pendular vibrations. Applying this consideration to wind instruments, and bearing in mind the initial pressure caused by the escape 
of air from the lips, it would appear probable that mouthpieces of different forms so modify this initial pressure as to cause a variety in the number and intensity of the upper partial tones.

XVII. On the Nebular Hypothesis.-IX. Radiation and Rotation. By Pliny Earle Chase, LL.D., S.P.A.S., Professor of Philosophy in Haverford College*.

[Continued from vol. v. p. 367.]

A MONG the most interesting of the unsolved astronomical A problems are the questions as to the origin of solar radiation and of cosmical rotation. These two problems, as I have already shown, are intimately connected, at the centre of our system, by the ultimate equality which exists between the velocity of light, the limiting centrifugal velocity of solar rotation, and the velocity of complete solar dissociation.

It has been commonly assumed that physical forces tend to ultimate equilibrium and consequent complete stagnation. The imperfections of any plan which looks to such a final result have led some writers to suppose that there may be some compensating provisions, hitherto undiscovered, for a renewal of activity. In the search for such provisions, the equality of action and reaction, and the possibility that the compensation is continually furnished by Him who is ever " upholding all things by the word of his power," seem to have been wholly overlooked.

If we assume the existence of a luminiferous æther, whether as a reality, or as a convenient representative of coordinated central forces, its undulations, when obstructed by inert centres, would necessarily lead to such phenomena as those of gravitation, light, heat, electricity, magnetism, \&c. Confining ourselves for the present to the action of gravitation, it is well known that the limiting velocity of possible gravitating action and consequent centrifugal reaction at any given point is $\sqrt{\overline{2} g r}$, the velocity varying as $\sqrt{\frac{1}{r}}$. If, according to the hypothesis of Mossotti, each particle is provided with a definite æethereal atmosphere, the density of that atmosphere in a condensing nucleus should vary as $\frac{1}{r^{3}}$. But, according to Graham's law,

* Communicated by the Author, having been read before the American Philosophical Society, June 21, 1878. 